\title{
Metamaterial Transparency Induced by Cooperative Electromagnetic Interactions
}

\author{
Stewart D. Jenkins and Janne Ruostekoski \\ Mathematical Sciences and Centre for Photonic Metamaterials, University of Southampton, Southampton SO17 1BJ, United Kingdom
} (Received 14 May 2013; revised manuscript received 21 August 2013; published 30 September 2013)

\begin{abstract}
We propose a cooperative asymmetry-induced transparency, CAIT, formed by collective excitations in metamaterial arrays of discrete resonators. CAIT can display a sharp transmission resonance even when the constituent resonators individually exhibit broad resonances. We further show how dynamically reconfiguring the metamaterial allows one to actively control the transparency. While reminiscent of electromagnetically induced transparency, which can be described by independent emitters, CAIT relies on a cooperative response resulting from strong radiative couplings between the resonators.
\end{abstract}

Electromagnetically induced transparency (EIT), a result of destructive interference between different excitation paths, causes an otherwise opaque collection of electromagnetic (EM) emitters to become transparent over a range of frequencies. In atomic gases, interference between atomic level transitions prevents the excitation of a transition that scatters incident light [1-3]. This interference abruptly alters the dispersion relation for frequencies in the transparency window, providing a mechanism to slow [4] or even stop light for later retrieval [5,6]. Slow and stopped light pulses have lead to applications in sensitive magnetometry [7-10], all-optical switching [11], and quantum memories [12-15].

Several theoretical proposals [16-19] and experimental realizations [20-29] have transferred the idea of EIT in independently scattering atoms to metamaterial arrays of circuit elements. In these artificially structured materials, the unit-cell resonators (metamolecules) play a role analogous to atoms in conventional EIT. A transmission resonance forms via coupling between two modes of plasmonic excitations in independently scattering metamolecules: a bright mode that strongly radiates and a dark mode with a narrower radiative linewidth. However, if the two modes have comparably broad linewidths and the metamolecules scatter independently, the quality of EIT-like transmission resonances is severely limited.

Recent studies [30-34] have shown that, rather than independently, certain systems of closely spaced resonators respond cooperatively to an incident field. In particular, interactions between resonators that are mediated by scattered EM fields result in collective modes of resonator excitations [30,35], several of which have significantly narrowed radiative linewidths.

In this Letter, we show how to exploit such collective modes to realize a cooperative transmission resonance. We propose a cooperative asymmetry-induced transparency (CAIT) in metamaterials. Unlike transmission resonances based on independent scatterers, the bright and dark modes in CAIT are collective. Specifically, the dark mode possesses a cooperatively narrowed resonance linewidth. This narrowing leads to a sharp resonance of high transmission, even though the resonators forming the metamaterial would individually, in isolation, exhibit broad resonances.

The transmission resonance is sensitive to the size of the system and the specific resonator configuration. Limited only by intrinsic nonradiative losses, the transmission resonance and group delay of a transmitted pulse can become progressively narrower and longer, respectively, with increasing size of a two-dimensional (2D) metamaterial array. In a $205 \times 205$ array, for example, we estimate that the resonance width (pulse delay) can reach approximately $\Gamma / 1000(1600 / \Gamma)$, where $\Gamma$ is the linewidth of a single isolated resonator. Furthermore, changing relative positions of the resonators alters the EM mediated interactions between them, and, hence, the cooperative material response. We show that using reconfigurable metamaterials [36,37], in which one can dynamically shift the layout of the metamolecules, allows one to actively control the transparency.

To illustrate CAIT, we consider a 2D array of asymmetric split rings (ASRs) [32,38], consisting of pairs of concentric circular arcs (Fig. 1). The setup is closely related to recent transmission resonance experiments [32]. In each ASR, currents can flow symmetrically, producing a net electric dipole along the direction $\hat{\mathbf{d}}$, or antisymmetrically, producing a net magnetic dipole along $\hat{\mathbf{m}}$. CAIT forms from the coupling between two phase-coherent collective modes of ASR excitations that are phase matched with an incident EM plane wave propagating perpendicular to the array. The incident field drives the phase-matched electric (PME) mode, dominated by all electric dipoles oscillating in phase, while the phase-matched magnetic (PMM) mode, consisting almost entirely of magnetic dipoles perpendicular to the array, does not directly couple to the incident field. In an array with subwavelength lattice spacing, magnetic dipole radiation emitted into the plane of the array repeatedly scatters off other resonators and can become trapped, causing the radiative linewidth of the PMM mode $\gamma_{M}$ to narrow with system size. 


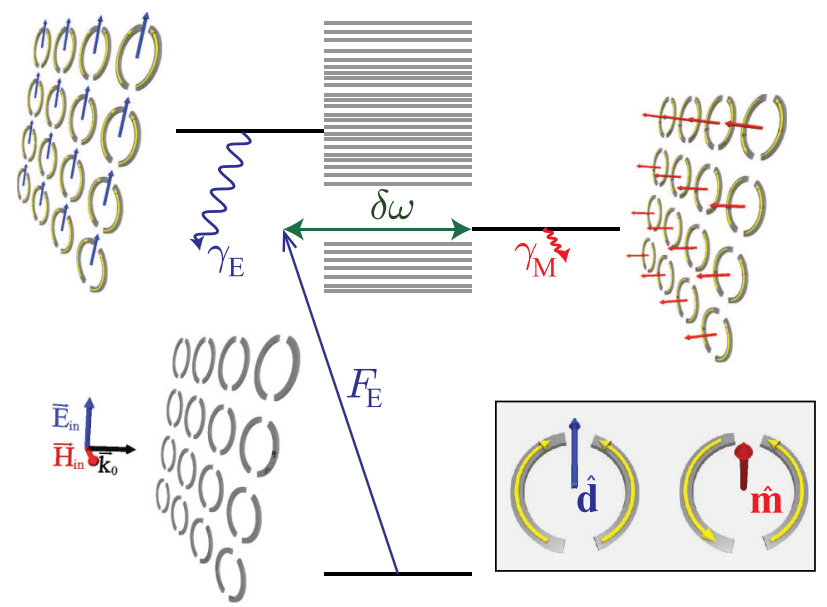

FIG. 1 (color online). A schematic illustration of CAIT in an array of ASRs. The inset shows symmetric (antisymmetric) currents in the ASR meta-atoms producing electric (magnetic) dipoles along $\hat{\mathbf{d}}(\hat{\mathbf{m}})$. The PME (PMM) mode is illustrated in the upper left (upper right), and has a decay rate $\gamma_{E}\left(\gamma_{M}\right)$. An incident wave couples an unexcited (lower) array to the PME mode with strength $F_{E}$, while asymmetry $\delta \omega$ induces a coupling between the PME and PMM modes. The grey lines represent other collective modes.

Because it radiates only weakly $[31,39]$, the PMM mode can be used as a collective dark mode in CAIT. For example, in a $33 \times 33$ array of split rings separated by half a wavelength, cooperative interactions reduce $\gamma_{M}$ 50-fold [30]. An excited superradiant PME mode, on the other hand, radiates with rate $\gamma_{E} \gtrsim \Gamma \gg \gamma_{M}$, scattering the field into the forward and backward directions and reflecting the incident field. We will show how an interference permits the excitation of the cooperatively narrowed PMM mode at the expense of the PME mode, forming a transmission resonance with an active control.

We consider a 2D square lattice of identical ASRs in the $z=0$ plane with subwavelength lattice spacing $a$ and lattice vectors $\mathbf{a}_{1}=a \hat{\mathbf{e}}_{x}$ and $\mathbf{a}_{2}=a \hat{\mathbf{e}}_{y}$. The ASR electric (magnetic) dipoles-produced by symmetric (antisymmetric) current oscillations-are oriented along $\hat{\mathbf{d}}=\hat{\mathbf{e}}_{y}\left(\hat{\mathbf{m}}=\hat{\mathbf{e}}_{z}\right)$ [39]; Fig. 1. Each ASR, labeled by index $\ell(\ell=1, \ldots, N)$ and centered at $\mathbf{r}_{\ell}$, comprises two meta-atoms (circular arcs). A meta-atom, labeled by index $j(j=1, \ldots, 2 N)$, behaves as a radiatively damped $L C$ circuit which is driven by the incident field and the fields emitted by all other meta-atoms in the system [30]. We describe the current flow in meta-atom $j$ by a slowly varying complex amplitude $b_{j}$ [39]. The meta-atom resonance frequencies are centered on $\omega_{0}$. Owing to an asymmetry in arc lengths, the resonance frequencies of the right $(j=2 \ell)$ and left $(j=2 \ell-1)$ meta-atoms in each ASR are shifted by $\delta \omega$ and $-\delta \omega$, respectively. The oscillating electric and magnetic dipoles of each meta-atom radiate at respective rates $\Gamma_{E}$ and $\Gamma_{M}$.
To better understand how a collection of ASRs behaves in concert, we first examine a single, isolated ASR of two interacting arcs. The dynamics of an ASR $\ell$ can be described by the amplitudes of symmetric, $c_{\ell,+}$, and antisymmetric, $c_{\ell,-}$, current oscillations, which are given in terms of the meta-atom variables as $c_{\ell, \pm}=$ $\left(b_{2 \ell} \pm b_{2 \ell-1}\right) / \sqrt{2}$. These oscillations are eigenmodes of a single symmetric split ring (SSR) $(\delta \omega=0)$ with the radiative decay rates $\gamma_{+} \approx 2 \Gamma_{E}$ and $\gamma_{-} \approx 2 \Gamma_{M}$ and resonance frequencies $\omega_{0} \pm \delta$.

In a single ASR, the asymmetry shifts the resonance frequencies of the left and right arcs. As a result, the symmetric and antisymmetric oscillations are no longer eigenmodes of a single ASR, and the evolution of those oscillations becomes coupled

$$
\dot{c}_{\ell, \pm}=\left(-\gamma_{ \pm} / 2 \mp i \delta\right) c_{\ell \pm}-i \delta \omega c_{\ell, \mp}+F_{\ell, \pm},
$$

where $F_{\ell,+} \simeq i e^{i \omega_{0} t}\left[6 \pi \epsilon_{0} c^{3} \Gamma_{E} / \omega_{0}^{3}\right]^{1 / 2} \hat{\mathbf{d}} \cdot \mathbf{E}_{\text {in }}^{+}\left(\mathbf{r}_{\ell}\right)$ and $F_{\ell,-} \simeq-e^{i \omega_{0} t}\left[6 \pi c^{3} \Gamma_{M} /\left(\mu_{0} \omega_{0}^{3}\right)\right]^{1 / 2} \hat{\mathbf{m}} \cdot \mathbf{B}_{\text {in }}^{+}\left(\mathbf{r}_{\ell}\right)$ represent the driving of the ASR electric and magnetic dipoles by positive frequency components of the incident electric $\mathbf{E}_{\text {in }}^{+}$ and magnetic $\mathbf{B}_{\text {in }}^{+}$fields. We will specifically consider the arrangement $\hat{\mathbf{m}} \cdot \mathbf{B}_{\text {in }}=0$. An EIT-like resonance of independently scattering ASRs requires that $\gamma_{\mp} \ll \gamma_{ \pm}$. This would allow the dark mode (with a lower emission rate) to be highly excited so that the coupling $\delta \omega$ to the bright mode (with a higher emission rate) destructively interferes with the driving of the bright mode by the incident field. In most experimental situations involving ASRs [32,38], however, $\gamma_{+}$and $\gamma_{-}$are comparable. Therefore, an array of independently scattering ASRs cannot exhibit an EITlike transmission resonance.

The situation differs, however, in a metamaterial array of several ASRs that interact via scattered EM fields. As a result of interactions, the system possesses collective modes of oscillation extended over the metamaterial. To show how CAIT can emerge from these collective modes, we construct an approximate phenomenological model from the PME and PMM modes, the two collective modes that are phase matched with the incident field. We use this model to analytically calculate the steady-state reflectance and transmittance. The mode properties, the accuracy of the phenomenological model, and the role of other collective modes in the metamaterial's EM response are numerically determined using the formalism introduced in Ref. [30]. These calculations fully incorporate all dependent scattering processes [30,40-45] between the resonators to all orders. Applying the formalism to a 2D array of ASRs [31] yielded a narrowing of collective linewidths with system size that agreed extremely well with experimental measurements of transmission resonances [32].

In the analysis, we approximate the incident EM field by $\mathbf{E}_{\text {in }}^{+}(\mathbf{r}, t)=\mathcal{E} \hat{\mathbf{e}}_{y} e^{i k z-i \Omega t} \quad(k=\Omega / c)$. The collective dynamics of the full metamaterial system, described by meta-atom variables $\mathrm{b} \equiv\left(b_{1}, b_{2}, \ldots, b_{2 N-1}, b_{2 N}\right)^{T}$, is governed by $[30,31]$ 


$$
\dot{\mathrm{b}}=\mathcal{C} \mathrm{b}+\mathrm{F}(t), \quad \mathcal{C}=\mathcal{C}_{\mathrm{SSR}}-i \delta \omega \mathcal{A} .
$$

In the radiative dynamics of the meta-atoms, described by $\mathcal{C}$, we separate the contributions of $\mathcal{C}_{\mathrm{SSR}}$ and $i \delta \omega \mathcal{A}$, so that the matrix $\mathcal{C}_{\mathrm{SSR}}$ describes the collective dynamics of the metamaterial in the absence of asymmetry (i.e., in an array where all ASRs are replaced by SSRs), and $\delta \omega \mathcal{A}$ accounts for the resonance shifts of the individual meta-atoms due to the asymmetry of the ASRs. The diagonal elements of the interaction matrix $\mathcal{C}_{\mathrm{SSR} j, j}=-\Gamma / 2$, where $\Gamma \equiv \Gamma_{E}+\Gamma_{M}$, represent decay rates and its off-diagonal elements account for interactions mediated by the scattered EM field, including near and far field contributions. The asymmetry in the ASRs shifts the meta-atom resonance frequencies by $\pm \delta \omega$. The sign of the frequency shift for a given metaatom is contained in the diagonal matrix $\mathcal{A}=$ $\operatorname{diag}(-1,1, \ldots,-1,1)$; the alternating signs of the elements indicate that the asymmetry shifts the frequencies of each side of the ASR in opposite directions. As a result of the incident wave, each element $j$ also experiences a driving $F_{j}=F_{0} \exp \left[i\left(k z_{j}-\Delta t\right)\right], \Delta \equiv \Omega-\omega_{0}$, with uniform amplitude $F_{0}$.

In the following analysis, it is beneficial to consider the collective modes that are eigenvectors of $\mathcal{C}_{\text {SSR }}$, i.e., eigenmodes of a metamaterial in the absence of metamolecule asymmetries. Of particular interest among the collective modes are the PME and PMM modes with phase-coherent electric and magnetic dipole excitations, respectively. The incident wave, whose electric field is parallel to the ASR electric dipoles, drives the PME mode. Since the incident wave's magnetic field is perpendicular to the ASR magnetic dipoles, the PMM mode is not directly driven. The asymmetry, however, couples the collective modes to each other in a way similar to how it couples the symmetric and antisymmetric oscillations of a single isolated ASR [39]. The phases and amplitudes of the electric dipoles in the PME mode closely match those of the magnetic dipoles in the PMM mode. Because of this mode matching, the asymmetry couples the PME and PMM modes more strongly to each other than to any other mode in the system. Therefore, we initially ignore the coupling of other collective modes to the PME and PMM modes. (This is later justified by the full numerical calculation and in [39].) The dynamics is, therefore, approximated by

$$
\begin{aligned}
\dot{c}_{E} & =\left(-i \delta_{E}-\gamma_{E} / 2\right) c_{E}-i \delta \omega c_{M}+f_{E}, \\
\dot{c}_{M} & =\left(-i \delta_{M}-\gamma_{M} / 2\right) c_{M}-i \delta \omega c_{E},
\end{aligned}
$$

where the subscripts $E$ and $M$ refer to PME and PMM modes, respectively (excitation amplitudes $c_{E, M}$, resonance frequency shifts $\delta_{E, M}$, decay rates $\gamma_{E, M}$, and driving $f_{E}$ ). Equations (3a) and (3b) are similar to those that describe the dynamics of atomic coherences in EIT [3]. Namely, when the system is driven on resonance with the PMM mode and $(\delta \omega)^{2} \gg \gamma_{M} \gamma_{E}$, the PMM mode is excited and the asymmetry induced coupling between the PMM and
PME destructively interferes with the driving of the PME mode to prevent its excitation.

In the calculation of the transmittance and reflectance we consider the field scattered from the resonators in the forward, $\hat{\mathbf{e}}_{z}$, and backward, $-\hat{\mathbf{e}}_{z}$, directions in the far field. We assume an absorbing planar barrier is placed around the metamaterial array so that the incident field can propagate through the array, but not around it, yielding the diffracted far field component of the incident field in the forward direction, $E_{I} \equiv \hat{\mathbf{d}} \cdot \mathbf{E}_{I}\left(\hat{\mathbf{e}}_{z}\right)$ [39]. Both the incident and the scattered fields $\mathbf{E}_{S}\left( \pm \hat{\mathbf{e}}_{z}\right)$ are polarized along the meta-atom electric dipoles. Therefore, we define the transmittance and reflectance amplitudes as $T=\left[E_{I}+\hat{\mathbf{d}} \cdot \mathbf{E}_{S}\left(\hat{\mathbf{e}}_{z}\right)\right] / E_{I}$ and $R=\hat{\mathbf{d}} \cdot \mathbf{E}_{S}\left(-\hat{\mathbf{e}}_{z}\right) / E_{I}[39]$.

We first estimate $R$ and $T$ in a phenomenological model by solving the steady-state response of Eqs. (3) and assuming a uniformly excited array. This simplified approach is then compared with a full numerical solution of Eq. (2) that incorporates all collective modes and the finite-size effects. In the phenomenological uniform mode approximation [39], we find

$$
R=\frac{R_{0} \gamma_{E} / 2\left[\gamma_{M} / 2-i\left(\Delta-\delta_{M}\right)\right]}{(\delta \omega)^{2}-\left(\Delta-\delta_{E}+i \gamma_{E} / 2\right)\left(\Delta-\delta_{M}+i \gamma_{M} / 2\right)}
$$

and $T=1+R$, where $R_{0}=-3\left(\Gamma_{E} / \gamma_{E}\right) /\left[2 \pi(a / \lambda)^{2}\right]$ is the reflectance of the system on resonance with the PME mode when $\delta \omega=0$, and $\lambda \equiv c /\left(2 \pi \omega_{0}\right)$. The phenomenological model (4) depends on the parameters of the collective modes PME and PMM, $\gamma_{E, M}$ and $\delta_{E, M}$, that may be calculated numerically [39]. Some example values are given in Table I. To illustrate the cooperative nature of CAIT, we here examine the transmission properties of three different sized arrays: a small $(11 \times 11)$, medium $(41 \times 41)$, and large $(205 \times 205)$. All have lattice spacing $a=0.4 \lambda, \Gamma_{E}=\Gamma_{M}$, and are composed of ASRs whose meta-atoms are separated by $u=0.18 \lambda$.

Figure 2 shows that, in the medium array, the uniform mode approximation reproduces the qualitative behavior of the full model [Eq. (2)]. This correspondence indicates that the PME and PMM modes play the dominant role in governing the array's transmission properties. The discrepancy arises due to finite-size effects in the full model, which, for example, allow the excitation of modes other than the PME and PMM modes.

TABLE I. The ASR asymmetries and the PME and PMM mode properties of the 2D ASR arrays used to demonstrate CAIT. The linewidth $\gamma_{M}$ varies inversely with the size of the array.

\begin{tabular}{lccccc}
\hline \hline Array size & $\delta \omega / \Gamma$ & $\delta_{E} / \Gamma$ & $\gamma_{E} / \Gamma$ & $\delta_{M} / \Gamma$ & $\gamma_{M} / \Gamma$ \\
\hline Small: $11 \times 11$ & 0.1 & 0.76 & 1.5 & 0.57 & 0.034 \\
Medium: $41 \times 41$ & 0.1 & 0.79 & 1.5 & 0.56 & $3.0 \times 10^{-3}$ \\
Large: $205 \times 205$ & 0.02 & 0.79 & 1.5 & 0.56 & $1.2 \times 10^{-4}$ \\
\hline \hline
\end{tabular}



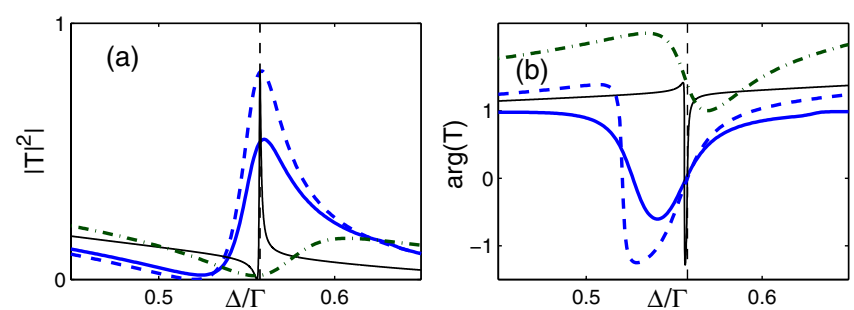

FIG. 2 (color online). (a) The intensity transmittance $|T|^{2}$ and (b) the phase delay $\arg (T)$ of the small ASR array in the full model (dot-dashed green line), the medium array in the full model (solid blue line), the medium array in the uniform mode approximation (dashed blue line), and the large array in the uniform mode approximation (thin black line). Simultaneously increasing the array size and reducing the asymmetry narrows the CAIT resonance.

Comparing the transmission spectra in Fig. 2, one finds that the medium and large arrays support CAIT, while the small array does not. When $\gamma_{M} \ll(\delta \omega)^{2} / \gamma_{E}$, as in the medium and large arrays, excitation of the PME mode is suppressed in a range of frequencies around the PMM resonance [39]. This suppression reduces reflection, opening a transparency window. Equation (4) indicates that resonant driving of the PMM maximizes the intensity transmittance when $\left|\delta_{E}-\delta_{M}\right| \ll \gamma_{E}$. The quality, or inverse spectral width, of the resonance increases in proportion to $(\delta \omega)^{-2}$ [39]. But the condition $\gamma_{M} \ll(\delta \omega)^{2} / \gamma_{E}$ imposes an upper bound on the achievable quality, as illustrated by the lack of a transparency window for the small array.

In contrast to EIT, the decay rate of the cooperative dark mode asymptotically scales as $\gamma_{M} \sim 1 / N$ with the number $N$ of ASRs [31]. This permits one to narrow the transparency window by designing an array with a greater $N$ and reduced $\delta \omega$, even when the constituent resonators individually would exhibit broad linewidths. On PMM resonance, Eq. (4) implies that the minimum asymmetry required to suppress $R$ below a given level $\delta \omega_{\min } \propto \sqrt{\gamma_{M}}$ [39]. Hence, the maximum attainable quality factor $\left[\propto(\delta \omega)^{-2}\right]$ of the transparency window increases in proportion to $N$ and is eventually only limited by nonradiative losses, resulting in very sharp resonances with high modulation depths. For example, from the asymptotic expressions of $\gamma_{E, M}$ and $\delta_{E, M}$ [39], we can deduce that simultaneously quintupling the side lengths of an array and reducing $\delta \omega$ by a factor of five narrows the resonance from about $\Gamma / 40$ to $\Gamma / 1000$, while maintaining the peak transmittance (Fig. 2).

The sharp transmission resonance exhibits a considerable sensitivity of phase delay $\varphi(\Delta) \equiv \arg [T(\Delta)]$ to $\Delta$. According to numerics, a pulse resonant on the PMM mode passing through the $41 \times 41$ sample would experience a group delay of $\tau_{g} \equiv d \varphi /\left.d \Delta\right|_{\Delta=\delta_{M}} \approx 47 / \Gamma$. The delay is further enhanced in the large array owing to
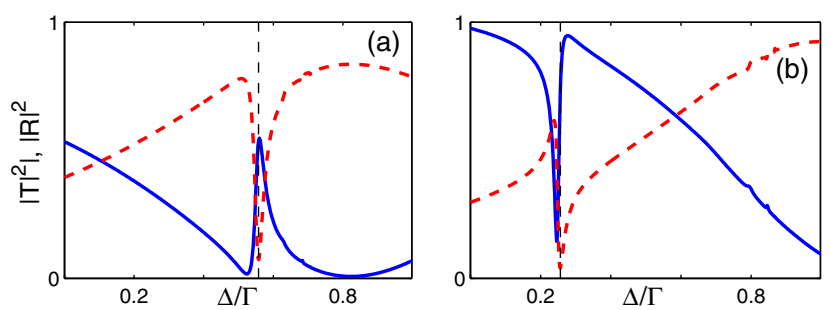

FIG. 3 (color online). The effect of reconfiguring the metamaterial geometry on the transmission resonance. The intensity transmittance (solid line) and intensity reflectance (dashed line) are calculated for a (a) $41 \times 412 \mathrm{D}$ array and (b) a lattice in which the two sublattices are shifted by $\delta \mathbf{R}=0.1 \hat{\mathbf{e}}_{z}$. Calculations account for excitation of and scattering from all collective modes. The vertical dashed lines indicate the PMM resonance.

linewidth narrowing and we estimate $\tau_{g} \approx 1600 / \Gamma$ in the phenomenological model.

Dynamically reconfiguring the metamaterial geometry $[36,37]$ provides an active control mechanism for the transparency. To illustrate this, we split the medium array into two interleaved sublattices with lattice vectors $\mathbf{a}_{1}=2 a \hat{\mathbf{e}}_{x}$ and $\mathbf{a}_{2}=a \hat{\mathbf{e}}_{y}$. The lattices are displaced from one and other by $a \hat{\mathbf{e}}_{x}+\delta \mathbf{R}$ so that for $\delta \mathbf{R}=0$, the ASRs form a square lattice. Figure 3 shows how distorting the lattice alters the transmission resonance. Displacing the sublattices by $\delta \mathbf{R}=-0.1 \lambda \hat{\mathbf{e}}_{z}$ generates a relative shift of about $0.5 \Gamma$ between the PME and PMM resonances, almost entirely eliminating the transparency. A fast control of metamaterial arrays [37], together with the sensitivity of cooperative resonances to the specific resonator configuration, could potentially open possibilities for stopped pulse and light storage applications [5].

In conclusion, we proposed a controllable mechanism to produce a cooperative transmission resonance CAIT. Whereas standard EIT can be described by independent emitters, CAIT relies on a cooperative response of the metamaterial. A transmission resonance forms when a subradiant collective mode, acting as a dark state, is excited at the expense of the mode that most efficiently couples to an incident EM field. Since the lifetime of the dark PMM mode increases with size of the array [31], the attainable quality of the resonance scales in proportion with the number of resonators in the metamaterial. For large arrays, only nonradiative decay, which could be incorporated into the analysis using a phenomenological parameter [31], limits the attainable quality factor of the resonance. In low-loss materials, such as superconducting metamaterial arrays [46-51], the nonradiative decay, however, can be suppressed.

This work was supported by the EPSRC and the Leverhulme Trust. We would like to thank N. Papasimakis, V. Fedotov, V. Savinov, E. Plum, M. D. Lee, M. O. Borgh, and N. I. Zheludev for discussions. 
[1] K. J. Boller, A. Imamoglu, and S. E. Harris, Phys. Rev. Lett. 66, 2593 (1991).

[2] S. E. Harris, Phys. Today 50, No. 7, 36 (1997).

[3] M. Fleischhauer, A. Imamoglu, and J. P. Marangos, Rev. Mod. Phys. 77, 633 (2005).

[4] L. V. Hau, S.E. Harris, Z. Dutton, and C.H. Behroozi, Nature (London) 397, 594 (1999).

[5] C. Liu, Z. Dutton, C. H. Behroozi, and L. H. Hau, Nature (London) 409, 490 (2001).

[6] M. D. Lukin and A. Imamoglu, Nature (London) 413, 273 (2001).

[7] M. Fleischhauer and M. O. Scully, Phys. Rev. A 49, 1973 (1994).

[8] G. Katsoprinakis, D. Petrosyan, and I. K. Kominis, Phys. Rev. Lett. 97, 230801 (2006).

[9] D. Budker and M. Romalis, Nat. Phys. 3, 227 (2007).

[10] V. I. Yudin, A. V. Taichenachev, Y.O. Dudin, V.L. Velichansky, A.S. Zibrov, and S. A. Zibrov, Phys. Rev. A 82, 033807 (2010).

[11] A. Bahrami, A. Rostami, F. Nazari, and K. Abbasian, J. Mod. Opt. 57, 2021 (2010).

[12] M. Fleischhauer and M. D. Lukin, Phys. Rev. Lett. 84, 5094 (2000).

[13] T. Chanelière, D. N. Matsukevich, S. D. Jenkins, S.-Y. Lan, T. A. B. Kennedy, and A. Kuzmich, Nature (London) 438, 833 (2005).

[14] K. S. Choi, H. Deng, J. Laurat, and H. J. Kimble, Nature (London) 452, 67 (2008).

[15] S. D. Jenkins, Y. O. Dudin, R. Zhao, D. N. Matsukevich, A. Kuzmich, and T. A. B. Kennedy, J. Phys. B 45, 124006 (2012).

[16] S. Zhang, D. A. Genov, Y. Wang, M. Liu, and X. Zhang, Phys. Rev. Lett. 101, 047401 (2008).

[17] P. Tassin, L. Zhang, T. Koschny, E. N. Economou, and C. M. Soukoulis, Phys. Rev. Lett. 102, 053901 (2009).

[18] H. Lu, X. Liu, and D. Mao, Phys. Rev. A 85, 053803 (2012).

[19] L. Verslegers, Z. Yu, Z. Ruan, P. B. Catrysse, and S. Fan, Phys. Rev. Lett. 108, 083902 (2012).

[20] N. Papasimakis, V. A. Fedotov, N. I. Zheludev, and S. L. Prosvirnin, Phys. Rev. Lett. 101, 253903 (2008).

[21] N. Papasimakis, Y. H. Fu, V. A. Fedotov, S. L. Prosvirnin, D. P. Tsai, and N. I. Zheludev, Appl. Phys. Lett. 94, 211902 (2009).

[22] N. Liu, L. Langguth, T. Weiss, J. Kästel, M. Fleischhauer, T. Pfau, and H. Giessen, Nat. Mater. 8, 758 (2009).

[23] N. Liu, T. Weiss, M. Mesch, L. Langguth, U. Eigenthaler, M. Hirscher, C. Sonnichsen, and H. Giessen, Nano Lett. 10, 1103 (2010)

[24] L. Zhang, P. Tassin, T. Koschny, C. Kurter, S. M. Anlage, and C. M. Soukoulis, Appl. Phys. Lett. 97, 241904 (2010).

[25] J. Zhang, S. Xiao, C. Jeppesen, A. Kristensen, and N. A. Mortensen, Opt. Express 18, 17187 (2010).

[26] B. Luk'yanchuck, N. I. Zheludev, S. A. Maier, N. J. Halas, P. Nordlander, H. Giessen, and C. T. Chong, Nat. Mater. 9, 707 (2010).

[27] J. Zhang, W. Bai, L. Cai, Y. Xu, G. Song, and Q. Gan, Appl. Phys. Lett. 99, 181120 (2011).
[28] C. Kurter, P. Tassin, L. Zhang, T. Koschny, A. P. Zhuravel, A. V. Ustinov, S. M. Anlage, and C. M. Soukoulis, Phys. Rev. Lett. 107, 043901 (2011).

[29] P. Tassin, L. Zhang, R. Zhao, A. Jain, T. Koschny, and C.M. Soukoulis, Phys. Rev. Lett. 109, 187401 (2012).

[30] S. D. Jenkins and J. Ruostekoski, Phys. Rev. B 86, 085116 (2012).

[31] S. D. Jenkins and J. Ruostekoski, New J. Phys. 14, 103003 (2012).

[32] V.A. Fedotov, N. Papasimakis, E. Plum, A. Bitzer, M. Walther, P. Kuo, D. P. Tsai, and N. I. Zheludev, Phys. Rev. Lett. 104, 223901 (2010).

[33] T. S. Kao, S.D. Jenkins, J. Ruostekoski, and N.I. Zheludev, Phys. Rev. Lett. 106, 085501 (2011).

[34] G. Adamo, J. Y. Ou, J. K. So, S. D. Jenkins, F. De Angelis, K. F. MacDonald, E. Di Fabrizio, J. Ruostekoski, and N. I. Zheludev, Phys. Rev. Lett. 109, 217401 (2012).

[35] M. M. Burns, J. M. Fournier, and J. A. Golovchenko, Phys. Rev. Lett. 63, 1233 (1989).

[36] I. M. Pryce, K. Aydin, Y. A. Kelaita, R. M. Briggs, and H. A. Atwater, Nano Lett. 10, 4222 (2010).

[37] J. Y. Ou, E. Plum, J. Zhang, and N. I. Zheludev, Nat. Nanotechnol. 8, 252 (2013).

[38] V. A. Fedotov, M. Rose, S. L. Prosvirnin, N. Papasimakis, and N.I. Zheludev, Phys. Rev. Lett. 99, 147401 (2007).

[39] See Supplemental Material at http://link.aps.org/ supplemental/10.1103/PhysRevLett.111.147401 for technical details.

[40] B. A. van Tiggelen, A. Lagendijk, and A. Tip, J. Phys. Condens. Matter 2, 7653 (1990).

[41] O. Morice, Y. Castin, and J. Dalibard, Phys. Rev. A 51, 3896 (1995)

[42] J. Ruostekoski and J. Javanainen, Phys. Rev. A 55, 513 (1997).

[43] J. Ruostekoski and J. Javanainen, Phys. Rev. A 56, 2056 (1997).

[44] J. Javanainen, J. Ruostekoski, B. Vestergaard, and M. R. Francis, Phys. Rev. A 59, 649 (1999).

[45] S. D. Jenkins and J. Ruostekoski, Phys. Rev. A 86, 031602 (R) (2012).

[46] M. Ricci, N. Orloff, and S. M. Anlage, Appl. Phys. Lett. 87, 034102 (2005).

[47] N. Lazarides and G. P. Tsironis, Appl. Phys. Lett. 90, 163501 (2007).

[48] C. Du, H. Chen, and S. Li, J. Phys. Condens. Matter 20, 345220 (2008).

[49] J. Gu, R. Singh, Z. Tian, W. Cao, Q. Xing, M. He, J. W. Zhang, J. Han, H.-T. Chen, and W. Zhang, Appl. Phys. Lett. 97, 071102 (2010).

[50] A. L. Rakhmanov, V. A. Yampol'skii, J.A. Fan, F. Capasso, and F. Nori, Phys. Rev. B 81, 075101 (2010).

[51] V. Savinov, A. Tsiatmas, A. R. Buckingham, V. A. Fedotov, P. A. J. de Groot, and N. I. Zheludev, Sci. Rep. 2, 450 (2012). 